ÉGYPTE monde arabe

\section{Égypte/Monde arabe}

$24 \mid 1995$

Anthropologies de l'Égypte 1

\title{
Des Nubies, des Nubiens : traditions scientifiques et locales de l'identité
}

\section{Frédérique Fogel}

\section{(2) OpenEdition \\ Journals}

Édition électronique

URL : https://journals.openedition.org/ema/634

DOI : $10.4000 /$ ema.634

ISSN : 2090-7273

\section{Éditeur}

CEDEJ - Centre d'études et de documentation économiques juridiques et sociales

\section{Édition imprimée}

Date de publication : 31 décembre 1995

Pagination : 75-86

ISSN : 1110-5097

\section{Référence électronique}

Frédérique Fogel, «Des Nubies, des Nubiens : traditions scientifiques et locales de l'identité », Égypte/ Monde arabe [En ligne], 24 | 1995, mis en ligne le 08 juillet 2008, consulté le 07 juillet 2022. URL : http:// journals.openedition.org/ema/634; DOl : https://doi.org/10.4000/ema.634

Ce document a été généré automatiquement le 7 juillet 2022.

Tous droits réservés 


\title{
Des Nubies, des Nubiens : traditions scientifiques et locales de l'identité
}

\author{
Frédérique Fogel
}

1 Chaque discipline participant des Études nubiennes présente une vision particulière de la Nubie, dont certains thèmes sont communs à l'ethnohistoire nubienne ${ }^{1}$. Une des propositions de l'analyse anthropologique consiste à mettre au jour des articulations entre tradition scientifique et tradition locale: en examinant les relations entre histoire et ethnohistoire - tous les mythes ne se référant pas seulement au second terme de la comparaison -, on interroge ici quelques aspects de l'attribution et de la revendication d'une identité, en commençant par qualifier la démarche des nubiologues.

2 La Nubie en tant qu'objet d'étude n'a pas plus de cent ans. Les archéologues se sont intéressés les premiers à cette région qui s'étend le long du Nil entre Aswan (Égypte) et Dongola (Soudan). Voie de passage et trait d'union entre l'Afrique et les civilisations de la Méditerranée et du Moyen-Orient pendant plus de cinq mille ans, et matière fantasmagorique de choix depuis Hérodote jusqu'aux voyageurs romantiques du XIX siècle, le lieu n'était pas inconnu. De nombreux documents hiéroglyphiques décrivent les relations souvent conflictuelles entre les anciens Égyptiens et les Nubiens, tour à tour esclaves et mercenaires du "pays de l'or» (noub) tant convoité ou ennemis redoutés du « pays de l'arc » (Ta-seti) (Valbelle, 1990 ; Midant-Reynes, 1992).

Deux travers importants ont marqué et continuent de marquer dans une large mesure la nubiologie. D'une part, et bien que de remarquables efforts aient été accomplis depuis la Campagne de l'Unesco (1959-69)², les données archéologiques présentent encore des lacunes et certaines périodes sont insuffisamment étudiées (Vercoutter, in Davis, 1991). De ce fait, l'orientation des travaux est généralement tributaire des questions suscitées par la tradition-mère, l'égyptologie : la Nubie n'existe bien souvent que «vue de l'Égypte» ou, plus récemment, «vue du Soudan", selon l'identité incertaine - voire marginale - que lui accorde l'archéologie égyptienne et nubiosoudanaise. Les difficultés d'interprétation tiennent souvent au rôle, réel ou supposé, de l'Égypte dans l'évolution culturelle de la Nubie. Très tôt engagée dans des échanges 
économiques et politiques souvent inégaux avec son puissant voisin du nord, cette dernière ne se voit concéder qu'une autonomie relative dans l'histoire, et l'écho de cette dépendance apparaît dans les études archéologiques comme une conséquence de l'histoire de la discipline sur les recherches.

D'autre part, l'évolution de la nubiologie se trouve liée au barrage d'Aswan, dont la première version, le «barrage des Anglais ", fut édifiée au tournant du siècle : chaque période d'investigation importante est motivée par l'imminence de travaux de rehaussement qui vont modifier le paysage par l'extension du lac de retenue, en 1911-12, en 1932-33 et enfin dans les années soixante, avec la construction du haut barrage. Ainsi, en 1907, a lieu le premier Archaeological Survey of Nubia (Reisner, 1910), suivi en 1930 d'une deuxième étude d'urgence (Sandford \& Arkell, 1933), puis de la Campagne de l'Unesco, point d'orgue de la série, qui comprend un volet ethnographique (Nubian Ethnological Survey) - mené par le Social Research Center de l'Université américaine du Caire (Fernea, 1966) - portant sur le mode de vie traditionnel des populations qui vont être transférées en 1963-64. Les deux disciplines ont donc en commun la particularité d'émerger dans une situation d'extrême urgence où leur objet se constitue en même temps qu'il disparaît.

Un objet mouvant : des difficultés pour l'identifier

Plusieurs obstacles rendent laborieuse l'identification de l'objet nubiologique, et en premier lieu l'usage assez confus du terme «nubien» qui renvoie souvent, dans les textes scientifiques, à deux concepts liés mais distincts: d'une part, comme terme ethnique, il désigne une population ou un groupe de populations; d'autre part, comme terme géographique, il indique la ou les région(s) que cette population ou ce groupe a occupée(s) ou occupe. La délimitation du pays varie ainsi selon les époques, les habitants et les commentateurs : les Nubiens seraient bien les habitants de «la » Nubie, mais les frontières se déplacent au cours de l'histoire presque autant que sa population qui, tantôt transporte son pays - et ses mythes d'origine - avec elle, tantôt abandonne sa «terre d'origine » qui devient alors un lieu d'ancrage mythologique lointain, tandis que le nouvel espace de vie ne se trouve pas investi de valeurs identitaires comparables. Pour rendre compte de l'espace nubien, il faudrait imaginer un «feuilletage » dont les plans superposés ne se recouvrent pas exactement, et ont même plutôt tendance à se décaler avec le temps, s'éloignant de plus en plus d'un « centre » lui-même mobile.

Du point de vue géographique, la Nubie s'étendait de part et d'autre du Nil Moyen : la frontière septentrionale était marquée, jusqu'à la mise en service du haut barrage, par la première cataracte qui coupe le fleuve au sud d'Aswan $\left(24^{\circ} \mathrm{N}\right)$ et dont la fonction s'est vue affaiblie par l'ouvrage hydraulique construit en amont. À l'est et à l'ouest, les limites étaient celles, rapidement atteintes, des terres cultivées. Les géographes distinguent une «basse » Nubie, zone morphologiquement homogène (grès de Nubie) jusqu'à la deuxième cataracte au sud de Wadi Halfa. Sur environ $350 \mathrm{~km}$, la vallée était étroite, les zones fertiles séparées par des affleurements rocheux (khor), le plateau aride atteignant souvent le lit du fleuve. La Basse Nubie, aujourd'hui engloutie, a longtemps été «la » Nubie des archéologues, Wawat dans les textes égyptiens. En amont de Wadi Halfa, l'environnement de la Haute Nubie (Kash, le Kouch biblique) est plus varié, les cataractes délimitent des zones de grès alternant avec des étendues de granit, comme le batn al-hagar ( "ventre de pierre ») entre la deuxième et la troisième cataracte.

7 Historiquement, les frontières de la Nubie se sont longtemps déplacées entre la première cataracte et le confluent des deux Nils selon, d'une part, les résultats des 
entreprises commerciales que menait l'Égypte dans cette région d'où provenaient l'or, l'ivoire et les esclaves, et, d'autre part, l'existence ou l'absence d'un État nubien. Dès l'Ancien Empire, la limite méridionale de l'Égypte est marquée par une stèle-frontière célébrant l'emprise de Pharaon sur les peuples de la région, ou par les forteresses construites entre l'île d'Éléphantine et la deuxième cataracte. Sous le Moyen Empire, la souveraineté de l'Égypte atteint Semna, et la stèle du même nom indique qu'«il est interdit à tout Nubien de descendre plus au nord, à pied comme en bateau, de même qu'aux troupeaux des Nubiens" (Valbelle, op. cit., p. 41); sous le Nouvel Empire, l'Égypte s'étend jusqu'à la quatrième cataracte; après l'effondrement du Nouvel Empire, les pharaons nubiens de Napata règnent sur l'Égypte.

8 Napata puis Méroé marquent l'«époque héroïque» de la Nubie (Adams, 1984) au tournant de notre ère. Le royaume kouchite se détache alors de l'influence égyptienne pour se tourner vers l'Afrique et profiter de sa situation au carrefour des voies caravanières entre la mer Rouge, l'Abyssinie et le Kordofan. La Basse Nubie est une fois de plus désertée, jusqu'à l'introduction de la saqia dans la vallée du Nil par les Romains (Trigger, 1965). La population de Haute Nubie s'accroît par l'immigration de groupes originaires des déserts environnants, et différents déplacements aboutissent à la formation de trois États - du nord au sud, Mâris, Makuria et Alodia - christianisés au VIe siècle.

9 Un siècle plus tard, des Arabes des tribus Rabî‘a et Juhayna, venant de la mer Rouge ou remontant le cours du Nil, commencent à s'installer en Nubie et provoquent plusieurs mouvements de migration. La Nubie chrétienne connaît encore quelques périodes de prospérité sous la protection d'une convention de non-agression (baqt) (Cuoq, 1986), puis s'étiole et disparaît au XIVe siècle à la suite des alliances des familles dirigeantes avec les tribus arabes (Ibn Khaldûn, 1987). L'arabisation et l'islamisation de la Nubie sont progressives, pondérées par la «nubianisation» des Arabes qui, notamment au nord et au sud de la région, adoptent les langues et les traditions culturelles indigènes. Les conséquences de ce double processus subsistent aujourd'hui dans les revendications d'ascendance, certains groupes nubiens d'Égypte, comme les Kenuz, ayant conservé leur langue tout en se réclamant d'une origine arabe tandis que d'autres, comme les Ja'aliyyin du Nord-Soudan, se sont arabisés au point qu'ils nient leur origine nubienne (Adams, 1984).

10 Car la Nubie n'est pas une zone linguistique homogène; différentes populations pratiquant des langues proches ont successivement ou parallèlement occupé des zones distinctes, et l'histoire de leurs déplacements et de leurs contacts n'est pas fermement établie. Des locuteurs nubiens, originaires du sud-ouest du Soudan, auraient immigré dans la vallée du Nil à l'époque méroïtique, puis se seraient divisés en deux groupes Danagla-Kenuz d'une part, et Mahas-Fadidja d'autre part - au Ille siècle. Sous diverses influences, notamment celle de tribus arabes, ces deux ethnies qui forment «la population » nubienne se sont étendues le long du Nil vers le nord et installées de part et d'autre de la deuxième cataracte en s'entrecroisant : soit, du sud au nord, Danagla, Mahas, Fadidja et Kenuz ${ }^{3}$. À l'époque moderne - jusqu'à la construction du haut barrage -, les locuteurs nubiens habitaient entre Aswan et le village soudanais de Debba (près de l'ancienne ville de Dongola, $18^{\circ} \mathrm{N}$ ), soit entre la première et la quatrième cataracte.

11 La frontière actuelle entre l'Égypte et le Soudan, tracée par le traité du Condominium le 19 janvier 1899, passe par le $22^{\circ} \mathrm{N}$ qui coupe le Nil au Jebel Sahaba, à $5 \mathrm{~km}$ au nord de 
Wadi Halfa. La deuxième cataracte est en quelque sorte une frontière "interne ", géographique, en partie historique, devenue politique et ethnique. Cette partition politique entre Nubiens égyptiens et Nubiens soudanais a redoublé et compliqué la partition ethnique. Les deux groupes nationaux relèvent de deux groupes ethniques apparentés. Chaque sous-groupe a développé avec ses voisins un certain type de relations en fonction de son origine ethnique et de sa langue. Pour les deux composantes du premier groupe cité, Danagla et Kenuz, les échanges sont plus difficiles du fait de la distance qui les sépare, mais l'origine commune appuyée sur la langue demeure une composante significative des représentations de l'identité.

Cette configuration du pays nubien, délimité par la première et la quatrième cataracte et peuplé par les deux ethnies, n'est plus. Cet espace, vidé de ses habitants en 1963-64 et submergé par les eaux du lac Nasser au début des années soixante-dix, s'appelle désormais officiellement " Ancienne Nubie », par rapport à " la » Nouvelle Nubie qui est dédoublée en deux zones d'aménagement planifié, celle de Kom Ombo en Égypte et celle de Khashm al-Girba au Soudan: on retrouve dans ces deux cas une appellation géographique déterminée par l'appartenance ethnique de ses nouveaux habitants.

Les populations déplacées, qui emploient à regret les qualificatifs d'«ancienne» (qadîm) et de «nouvelle " (gadîd), continuent de se référer globalement à «la » Nubie quand ils parlent de leur "pays d'origine ». Poussés à la comparaison, les Nubiens d'Égypte établiront une partition dans le temps en distinguant les Nubies d'avant et d'après le transfert, ou plus fréquemment dans l'espace, le haut barrage séparant albalad fôq («le pays d'en haut », l'Ancienne Nubie) et al-balad taht (« le pays d'en bas », la Nouvelle Nubie), ce qui pourrait aussi se traduire par «Haute » et «Basse » Nubie dans une acception différente, déplacée par rapport à celle des géographes. À la demande des Nubiens, les villages reconstruits ont gardé leur nom d'origine, suivi de "nouveau».

14 Côté soudanais, les villages ont perdu leur nom et sont désignés par des numéros. La ville de Wadi Halfa en Nubie ayant été engloutie, le nouveau centre administratif du périmètre de Khashm al-Girba prend, en 1963, le nom de Halfa al-Gadîda (« Nouvelle Halfa »). Le même nom sert aussi à désigner la ville reconstruite au début des années quatre-vingt à proximité de l'ancienne Wadi Halfa, sur le lac Nasser appelé «lac Nubie » du côté soudanais. Depuis, les différents projets (réalisés pour certains) de réimplantation des Nubiens - soudanais au sud du lac et égyptiens autour d'Abu Simbel et au nord -, portent donc l'appellation de «Nouvelle Nouvelle Nubie » ou de « Nouvelle Ancienne Nubie ».

15 Ainsi «la» Nubie s'est-elle déplacée et fragmentée : le terme désigne actuellement chacun des sites où vivent des communautés nubiennes. Seuls les émigrés urbains ne nomment pas «Nubie » les quartiers qu'ils habitent, ce qui peut laisser supposer que, contrairement à d'autres populations rurales urbanisées, ils n'investissent pas totalement leur lieu d'habitation et restent profondément liés au lieu dont ils sont originaires, sinon à leur lieu d'origine.

Un objet composite. Des différents objets de l'identité

16 C'est justement là, dans le manque de cohésion entre ces différents espaces, que se manifeste pour une grande part la question de l'identité des Nubiens. Il ne s'agit pas de traquer leur origine réelle: la brève présentation qui précède donne une image des difficultés auxquelles sont confrontées les recherches nubiologiques. Pour ne prendre qu'un seul exemple, la détermination des frontières entre l'Égypte et la Nubie sous 
l'Antiquité repose notamment sur l'étude des contacts entre les deux populations à travers leur culture matérielle, le propos étant de savoir quel processus l'emporte de l'égyptianisation des cultures nubiennes ou de la nubianisation des colons égyptiens installés en Nubie durant les périodes de prospérité. Dans le même temps, d'autres éléments - en particulier linguistiques, dont je n'ai donné qu'un court aperçu tendraient à déplacer la question sur un terrain beaucoup plus vaste, à l'échelle du Soudan, pour déterminer avec plus de précision quelles sont les populations que l'on peut appeler "nubiennes » à la même époque, et le moins que l'on puisse dire est qu'elles sont vraisemblablement plus nombreuses et plus diversifiées que les quelques groupes auxquels les Égyptiens ont accès.

17 Les mouvements de population opèrent dans un espace trop large - dont n'apparaissent ici que des fragments - et sur une période trop longue pour que l'histoire de chaque groupe actuel soit reconstituée précisément, et les matériaux ne permettent pas d'aller au-delà d'un schéma global qui dessine des regroupements ponctuels de groupes différents - Napata, Méroé ou encore les royaumes chrétiens appelés à se défaire pour que s'organisent plus tard, et selon d'autres modalités, des configurations différentes. L'idée d'une "origine commune » est donc hypothétique, dans l'histoire comme dans l'ethnohistoire, car l'enjeu identitaire des Nubiens actuels n'est pas de reconstruire l'histoire pour faire émerger un groupe d'origine commun, un groupe de référence mais, à l'aide de différents éléments empruntés ou non à l'histoire, de construire une spécificité. Il serait même plus adéquat d'évoquer plusieurs identités se référant à plusieurs espaces car, à l'image d'une Nubie "feuilletée", répond celle d'une identité morcelée, différents mythes puisant dans des registres différents, voire contradictoires.

18 L'ethnohistoire nubienne applique à l'histoire de la région un traitement caractérisé par une manière spécifique de sélectionner des épisodes réels et de les mêler aux mythes, tant dans le domaine des relations avec les Égyptiens anciens et contemporains, que dans celui des rapports avec les " envahisseurs ", Égyptiens comme Arabes. Ce faisant, les Nubiens interprètent l'histoire à leur avantage, quitte à inventer une version locale fondée sur l'histoire des étrangers ou à attribuer les hauts faits qui animent leurs mythes à leurs propres ancêtres, qui viennent toujours d'ailleurs. En effet, la tendance générale dans la construction des mythes est un mouvement centripète qui permet de se situer à l'origine de toute chose, ou au plus près de l'origine des choses.

Prenons l'exemple de l'interprétation nubienne de l'Antiquité. L'histoire de l'Égypte antique vue par les Nubiens se fonde sur leur conviction d'être à l'origine du peuplement de la région, conviction qui se manifeste principalement sur trois modes. En premier lieu, leur situation géographique, en amont par rapport au reste du pays, leur donne une vision de l'antériorité orientée dans l'espace, un sens du temps, analogue au courant du Nil - leur propre histoire migratoire « coulerait » aussi du sud vers le nord -, et au sein duquel les fondateurs du pays originaires de l'amont répandent la "civilisation» vers l'aval. Évoquant les Égyptiens d'hier comme leurs ancêtres, les Nubiens égyptiens d'aujourd'hui se réfèrent aux monuments de l'Antiquité qui s'élevaient sur le "pays d'en haut » et dont les plus accessibles sont maintenant les temples de Philae et d'Abu Simbel, marquant symboliquement les frontières nord et sud de l'ancienne Basse Nubie. La présence de nombreux monuments sur leurs terres 
avant la construction du haut barrage constitue à leurs yeux la preuve matérielle de leur différence par rapport aux Égyptiens actuels.

À l'inscription de l'histoire dans le paysage, aux souvenirs des enfants d'alors qui jouaient dans les mines et des quelques étrangers qui venaient leur rendre visite, s'ajoute une mythologie basée sur la gloire des pharaons, que les Nubiens s'approprient d'autant plus aisément qu'elle leur permet de se situer à l'origine de l'histoire nationale tout en s'en démarquant. Se considérant comme les seuls véritables descendants de ces grands bâtisseurs, ils reprennent à leur compte les épisodes les plus glorieux de la période dynastique, sans se référer par ailleurs à une chronologie autre que celle qui rejette tous les ancêtres dans un lointain passé. L'idée générale d'une telle revendication ne tient pas d'une recomposition à partir de bribes de connaissances scolaires, mais plutôt de l'interprétation recentrée sur eux-mêmes d'un ensemble hétéroclite partagé par « tous » les Égyptiens.

21 En deuxième lieu, établissant un parallèle entre l'absence d'écriture de leur langue et la disparition de l'usage des hiéroglyphes, ils considèrent que ces derniers servaient à écrire le nubien, et que cette langue était donc celle des pharaons - la seule écriture nubienne connue à ce jour, mais non encore déchiffrée, date de la période méroïtique. Enfin, ils appuient leur certitude d'une différence physique les distinguant des Égyptiens sur une prétendue similitude de traits avec les pharaons tels qu'ils les imaginent d'après les représentations picturales et statuaires conservées dans les temples.

22 Autre exempte significatif du phénomène d'appropriation par les mythes, celui des relations entre les Kenuz - l'un des deux groupes nubiens égyptiens - et les Arabes. Au $\mathrm{VII}^{\mathrm{e}}$ siècle, des tribus originaires de la Péninsule arabique émigrent en Haute-Égypte. Une partie de la tribu dominante des Rabî'a, originaire du Nedj, s'assimile par des mariages aux Beja locaux. Leur nombre augmente, leur influence grandit, ils étendent leur pouvoir au moins de façon intermittente sur Aswan et la partie adjacente de la vallée du Nil. Sous les Fatimides, le leader de cette tribu combinée arabe-beja est accepté de facto comme gouverneur d'Aswan.

En 1006, le gouverneur Abû-l-Makârim livre au calife fatimide le prétendant umayyade Abû Rakwa. Pour ce service, il obtient le titre héréditaire de Kanz al-Dawfa (« trésor de la nation »). C'est cet événement qui amène la tribu dans son ensemble à être connue comme les Banû Kanz (Ban̂̂-l-Kanz), «les descendants du Kanz al-Dawla» (Adams, $1984)^{4}$.

24 Différents épisodes marquent les rapports conflictuels entre les Banû Kanz et le pouvoir égyptien. En 1172, l'expédition de Shams al-Dawla Tûrân Shâh aboutit à la mise à mort du Kanz al-Dawla et à l'expulsion des Banû Kanz d'Aswan. Certains se réfugient dans les collines de la mer Rouge; d'autres, plus nombreux, s'installent au nord de la Nubie. Ils s'intermarient avec la population locale, se nubianisent en partie linguistiquement et culturellement, et ils islamisent et arabisent - tribalisent - les Nubiens. Les Kenuz résulteraient de cet " amalgame ethnique », selon l'expression de Fernea (1966, p. 5).

L'épisode donne lieu à deux types de commentaires de la part des Kenuz. Soit les Banû Kanz sont des Arabes qui deviennent nubiens en adoptant la langue nubienne et en épousant des femmes nubiennes; en échange, les Nubiens reçoivent l'islam et la tribu, et les Kenuz considèrent les Banû Kanz comme des ancêtres parmi d'autres. Soit « Nos ancêtres étaient arabes; ils sont venus de la péninsule arabique ; ces grands guerriers ont été récompensés par le sultan pour avoir gagné une bataille; le souverain leur a 
donné un trésor [kanz]. » En dépit des apparences, ce récit ne joue pas dans l'esprit de ses promoteurs comme la preuve d'une origine étrangère - qui serait en outre la meilleure des origines possibles puisqu'elle mettrait en relation le groupe avec la terre de l'islam -, mais comme la démonstration de l'ancrage local des Kenuz face aux Fadidja, ces derniers étant demeurés à l'écart des influences arabes et ne pouvant revendiquer qu'une origine lointaine et non arabe, car ils ont été islamisés beaucoup plus tard par les Ottomans. Alors que la référence aux pharaons concerne l'ensemble des Nubiens vis-à-vis des Égyptiens, celle-là change d'échelle en opposant les deux groupes nubiens.

Dernier exemple, enfin, qui montre quels peuvent être les enjeux d'une distorsion de l'histoire dans le domaine religieux. L'islamisation a été, au nord de la Nubie, la conséquence d'une assimilation ethnique et politique promue par les tribus arabes dès le vII siècle, par les Banû Kanz auprès des Kenuz. Mais les Mehennâb, tribu kenzi, racontent leur islamisation autrement, en inscrivant à l'origine de leur généalogie en tant qu'ancêtre fondateur 'Abd al-Qâdir al-Gilânî, saint de Bagdad, descendant du Prophète et inspirateur de la confrérie Qâdiriyya - ce personnage étranger à la Nubie, dont les Mehennâb ne prétendent d'ailleurs pas qu'il y soit venu, est célébré pour ses "miracles» dans de nombreuses régions du monde musulman, notamment au Maghreb.

Comme pour nombre de tribus islamisées et, pour une part, arabisées, l'enjeu du récit d'origine est de légitimer leur identité par l'insertion de la généalogie tribale dans la généalogie globale de la communauté musulmane, la preuve de la meilleure des origines possibles étant l'appartenance à l'une des lignes de filiation qui remontent au Prophète et à ses Compagnons, et plus loin à 'Adnân ou à Kahtân, ancêtres de la généalogie arabe classique des tribus. Certaines tribus soudanaises revendiquent l'origine des Arabes qui les ont islamisées: ainsi, leur mythe d'origine rencontre l'histoire. Les Mehennâb auraient pu, à travers les Banû Kanz, se revendiquer des Rabî'a qui offraient une généalogie suffisamment prestigieuse. S'ils ont assimilé la structure de la généalogie musulmane, le contenu qu'ils Iui donnent n'est pas le plus simple : ils dénient l'histoire réelle de leur islamisation en rejetant toute trace de leurs relations avec la tribu arabe qui leur a donné le nom de Kenuz; en plaçant leur origine plus haut que celle des Banû Kanz, et en adoptant pour ancêtre fondateur un saint étranger, ils gomment délibérément leur participation à l'histoire régionale. Tout en remontant aussi haut dans la généalogie arabe, ils effacent de leurs origines tous les aspects latéraux qui les relient aux autres Nubiens. Ils font de leur "islamité » un critère de distinction à usage interne.

Autre conséquence de ce mythe, et non des moindres, sur la conception de l'espace des origines: dans le récit généalogique, des descendants de Gilâni venant de Bagdad s'étaient installés sur l'île d'Argo - île du batn ai-hagar où se sont effectivement réfugiés des Nubiens à la fin de la période méroïtique -, point de départ de la migration fondatrice conduite par l'ancêtre éponyme des Mehennâb jusqu'à l'Ancienne Nubie. Ce qui élargit d'autant la représentation indigène de la Nubie, espace considéré comme généré d'un point fondateur qui permet au groupe de revendiquer une origine méridionale encore plus lointaine.

Conclusion

29 L'argument développé ici se fonde sur un découpage, dans le temps et dans l'espace, de l'identité en tant que mode de représentation et de présentation de soi. Plusieurs 
thèmes reviennent, comme celui de l'orientation du paysage de la mémoire : le passé est associé à l'amont, au " haut ", à un sud lointain d'où viennent les ancêtres et à un sud plus proche - la terre d'avant le haut barrage - où sont demeurés les morts; au nord sont les étrangers, ceux dont la différence est irréductible, comme les Égyptiens et les Arabes, ou compréhensible lorsqu'il s'agit des groupes nubiens entre eux. La revendication de l'origine fonde une spécificité ethnique aux multiples facettes, dont le but est de se démarquer des autres.

En présentant l'objet composite fabriqué par l'ethnohistoire nubienne, j'ai choisi plusieurs aspects relatifs à l'imaginaire des relations entre les Nubiens et les pharaons, et entre les premiers et les Arabes des tribus, afin de souligner à la fois les modalités de construction de l'identité et le sens de ces élaborations. On remarque en effet que les interprétations locales ne cherchent pas simplement à utiliser des références externes pour situer chaque groupe dans un contexte global, elles ne construisent pas l'identité sur l'identique, mais orientent les éléments dont elles disposent de façon à centrer l'histoire sur le local. Pourquoi ce mouvement centripète? On serait tenté de répondre qu'il faut bien un sens aux tribulations d'une population migrante... Pourtant, il est manifeste qu'un processus de recentrage est bien à l'œuvre dans la construction de l'identité nubienne, recentrage qui semble en relation avec les déplacements successifs que les Nubiens ont connus - et continuent de connaître, car le haut barrage n'a pas interrompu les migrations. La distance temporelle entre les ancêtres fondateurs et leurs descendants actuels est réduite par les mythes qui se jouent de la profondeur historique comme de la chronologie. De même, la distance spatiale entre les différents lieux successivement occupés est réduite par le récit des migrations conduites par les ancêtres, ce qui est une façon d'intégrer les traumatismes liés aux déplacements en prétendant être à l'origine de ce que l'on subit.

En d'autres termes, interpréter l'histoire en la ramenant à soi, en se plaçant à l'origine, serait une manière de contrarier l'évidence de la dispersion et de la situation périphérique, voire marginale, que les Nubiens ont presque toujours occupée dans la région. Face à la tradition scientifique qui a construit un objet Nubie unique, mal défini, et assimilé les groupes différents en une seule population, les Nubiens opteraient pour une fragmentation très poussée au sein de laquelle chaque groupe édifie sa différence, donc son identité.

\section{BIBLIOGRAPHIE}

Adams William Y., Nubia, Corridor to Africa, Allen Lane, Princeton University Press (1st ed. 1977), Princeton, 1984.

Cuoq Joseph M., Islamisation de la Nubie chrétienne (Vlle-XVIe siècle) ; Bibliothèque d'études islamiques, vol. IX, Librairie orientaliste Paul Geuthner, Paris, 1986.

Davis W. V. (éd.), Egypt and Africa, Nubia from prehistory to Islam, British Museum Press - Egypt Exploration Society, Londres, 1991. 
Fernea Robert A. (ed.), Contemporary Egyptian Nubia, Human Relations Area Files Press lnc., 2 vol., New Haven, 1966.

Greenberg Joseph H., The Languages of Africa, Indiana University, The Hague, Mouton and Co, Bloomington, 1963.

Hassan Yusuf FadI, « Main Aspects of the Arab Migration of the Sudan », Arabica XIV, 1967, p. 14-23.

Ibn Khaldûn, Peuples et nations du monde [Extraits des 'Ibar traduits et présentés par Abdesselam Cheddadi], 2 vol., Sindbad, Paris, 1987.

MacMichael Harold A., A History of the Arabs in the Sudan, 2 vol., Frank Cass \& Co. Ltd. [1st ed. 1922], Londres, 1967.

Midant-Reynes Béatrix, Préhistoire de l'Égypte, des premiers hommes aux premiers pharaons, Armand Colin. Paris, 1992.

Reisner George Andrew, The Nubian Archaeological Survey Report for 1907-1908, National Printing Department, vol. 1, Le Caire, 1910.

Sandford K. S. \& Arkell W. J., Palaeolithic Man and the Nile Valley in Nubia and Upper Egypt, Chicago Oriental Institute [COI Publications 17], Chicago, 1933.

Säve-Söderbergh Torgny, Victoire en Nubie. La Campagne internationale de Sauvegarde d'Abou Simbel, de Philae et d'autres trésors culturels, Unesco, Paris, 1992.

Trigger Bruce G., History and Settlement in Lower Nubia, Yale University Press [Yale University Publication in Anthropology 69], New Haven, 1965.

Trimingham J. Spencer, Islam in the Sudan, Frank Cass and Co. [1st ed. 1949], Londres, 1965.

Valbelle Dominique, Les neuf arcs, l'égyptien et les étrangers de la préhistoire à la conquête d'Alexandrie, Armand Colin, Paris, 1990.

\section{NOTES}

1. Cet article développe certains thèmes abordés dans ma thèse : Migrations et identités d'un groupe nubien (Égypte), Université de Paris X, 1994.

2. Il s'agit de la campagne internationale de sauvetage des monuments de Nubie avant la submersion de la région par les eaux du lac Nasser (cf. notamment Säve-Söderbergh, 1992).

3. La discussion sur l'origine et l'évolution des langues nubiennes, classées Nile Nubian dans la famille Nilo-Saharan (Greenberg, 1963), n'est pas aboutie. Les Danagla et les Kenuz parlent la même langue, appelée dongolawi au Soudan et mattoki en Égypte ; la langue commune des Mahas et des Fadidja porte le nom des groupes qui la parlent, mahassi et fadidja.

4. Sur l'origine et l'histoire des Banû Kanz : MacMichael (1967, I, p. 149-151), Trimingham (1965, p. 68), Hassan (1967, p. 58-60). Ibn Khaldûn présente les Banû Kanz comme des descendants des Juhayna : « Dans le Sa'îd supérieur, d'Aswân à la terre de Nubie, et de là à l'Abyssinie, on trouve des tribus nombreuses et des clans dispersés, tous issus des Juhayna, une fraction des Qudâ'a. Ces tribus occupent toutes les terres désertiques de cette région, ont conquis les territoires des Nubiens et les ont soumis à leur joug ; elles ont empiété sur le pays des Abyssins et partagent avec eux les 
territoires limitrophes. Celles qui se sont fixées autour d'Aswân sont connues sous le nom d'Awlâd al-Kinz. Leur ancêtre, Kinz ad-Dawla, est resté célèbre par ses actions contre le gouvernement de l'Égypte. » (1987, II, p. 426)

INDEX

Mots-clés : identité, anthropologie, Nubie, nubiologie

\section{AUTEUR}

FRÉDÉRIQUE FOGEL

Université Paris X-Nanterre 